\title{
Smart aerosonde UAV longitudinal flight control system based on genetic algorithm
}

\author{
Ahmed Elbatal, Ahmed Medhat Youssef, Mohamed M. Elkhatib \\ Military Technical College, Cairo, Egypt
}

\section{Article Info}

Article history:

Received Mar 7, 2020

Revised Apr 29, 2021

Accepted Jul 14, 2021

\section{Keywords:}

Flight control system

Genetic algorithm

PID controller

UAV

\begin{abstract}
Synthesis of a flight control system for such an aircraft that achieves stable and acceptable performance across a specified flying envelope in the presence of uncertainties represents an attractive and challenging design problem. This study uses the genetic self-tuning PID algorithm to develop an intelligent flight control system for the aerosonde UAV model. To improve the system's transient responses, the gains of the PID controller are improved using a genetic algorithm (GA). Simulink/MATLAB software is used to model and simulate the proposed system. The proposed PID controller integrated with the GA is compared with the classical one. Three simulation scenarios are carried out. In the first scenario, and at normal conditions, the proposed controller performance is better than the classical one. While in the second scenario, identical results are achieved from both controllers. Finally, in the third scenario, the PID controller with GA shows the robustness and durability of the system compared with the classical PID in presence of external wind disturbance. The simulation results prove the system parameters optimization.
\end{abstract}

This is an open access article under the CC BY-SA license.

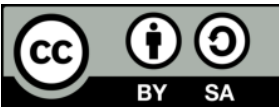

Corresponding Author:

Mohamed M. Elkhatib

Electronic Engineering Department

Military Technical College

Kobry Elkoba, Cairo, Egypt

Email: mohamed.m.elkhatib@ieee.org

\section{NOMENCLATURE}

$\varphi, \theta, \psi \quad$ Angular shifts in the roll, pitch, and yaw axes.

$\delta_{a}, \delta_{e}, \delta_{r}, \delta_{t h}$ Deflection of the aileron, elevator, rudder, and throttle, correspondingly.

$\mathrm{P}, \mathrm{q}, \mathrm{r} \quad$ Angular speed in the roll, pitch, and yaw axes

$\mathrm{u}, \mathrm{v}, \mathrm{w} \quad$ Roll, pitch, and yaw axis speed components, respectively

$h_{f}, h_{\text {ref }} \quad$ The current and desired altitudes

$v_{f}, v_{\text {ref }} \quad$ Airspeeds (current and target)

$S_{i}, C_{i} \quad$ Represents $\sin \theta$ and $\cos \theta$, respectively

\section{INTRODUCTION}

Unmanned aerial vehicles (UAVs) have become more popular in a variety of military and civilian applications where human participation is difficult or risky [1], [2]. The design of a UAV's flight control system to ensure robust stability and acceptable performance within a given flight envelope in the presence of actuator and sensor uncertainties, as well as external disturbances such as wind gusts, is one of the most difficult tasks that designers face [3]-[5]. Therefore, this paper is devoted to design a longitudinal flight control system for the fixed-wing aerosonde UAV. To achieve this objective, successive activities are 
considered for obtaining a mathematical model of the underlying aircraft, including the derivation of aircraft nonlinear equations of motion and tailoring them for use on the underlying aircraft. The dynamics of both sensors and actuators are integrated into the nonlinear model. Trimming the nonlinear model for steady-state flight conditions and obtaining the linearized models for these conditions are then considered. The longitudinal model is then extracted from the whole aircraft model.

Traditionally, classic PID controllers were utilized to create flight control systems, with ad-hoc approaches employed to adjust the controller gains in flight. This is a time-consuming and high-risk methodology [6], [7]. In this paper, the longitudinal flight control system is designed using a PID controller whose gains are optimally tuned by a genetic algorithm (GA) technique for suppressing the tracking errors and hence getting precision tracking. This significantly reduces the time and effort required to tune parameters, demonstrating that the era of the ad-hoc approach for tuning PID controllers is coming to an end. A comparative synthesis between the proposed genetic self-tuning PID and the classical PID controllers is performed for specific altitude and speed commands to demonstrate the proposed controller's capabilities and robustness in providing more acceptable responses in the influence of external wind disturbances.

\section{THE AEROSONDE MODEL}

\subsection{System block diagram and description}

Figure 1 shows aerosonde UAV model. The MATLAB aerosim block set is used to model the first transatlantic ocean UAV, aerosonde, as shown in Figure 2. By using the data of various test flights, the model of the aerosonde UAV is built up, as shown in Figure 2, which is utilized as a test model for aircraft [8]. The aerosim block set contains the tools of six degrees of freedom aircraft [9]. With the help of a $24 \mathrm{cc}, 1.2 \mathrm{~kW}$ engine and a payload of $1 \mathrm{~kg}$, the completely autonomous aerosonde UAV can reach a top speed of $40 \mathrm{~m} / \mathrm{s}$. With a height of up to 20,000 feet, the cruise speed is between 20 and $30 \mathrm{~m} / \mathrm{s}$. These specifications are for a $2.9 \mathrm{~m}$ wingspan and a $30 \mathrm{lb}$ weight. Although the control system of aerosonde is complicated, the ability to perform a mission is highly achievable due to its flexible design [10].

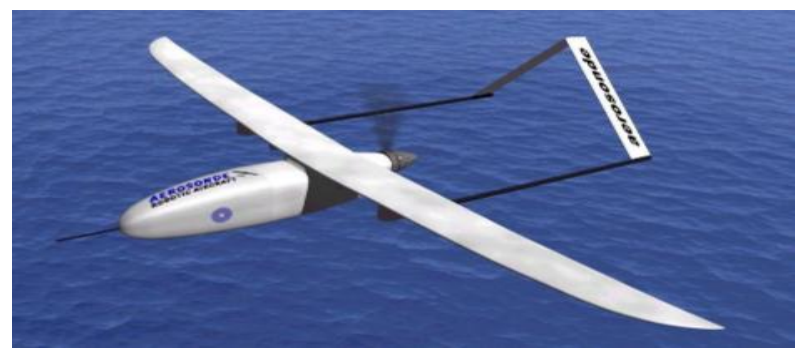

Figure 1. Aerosonde UAV model

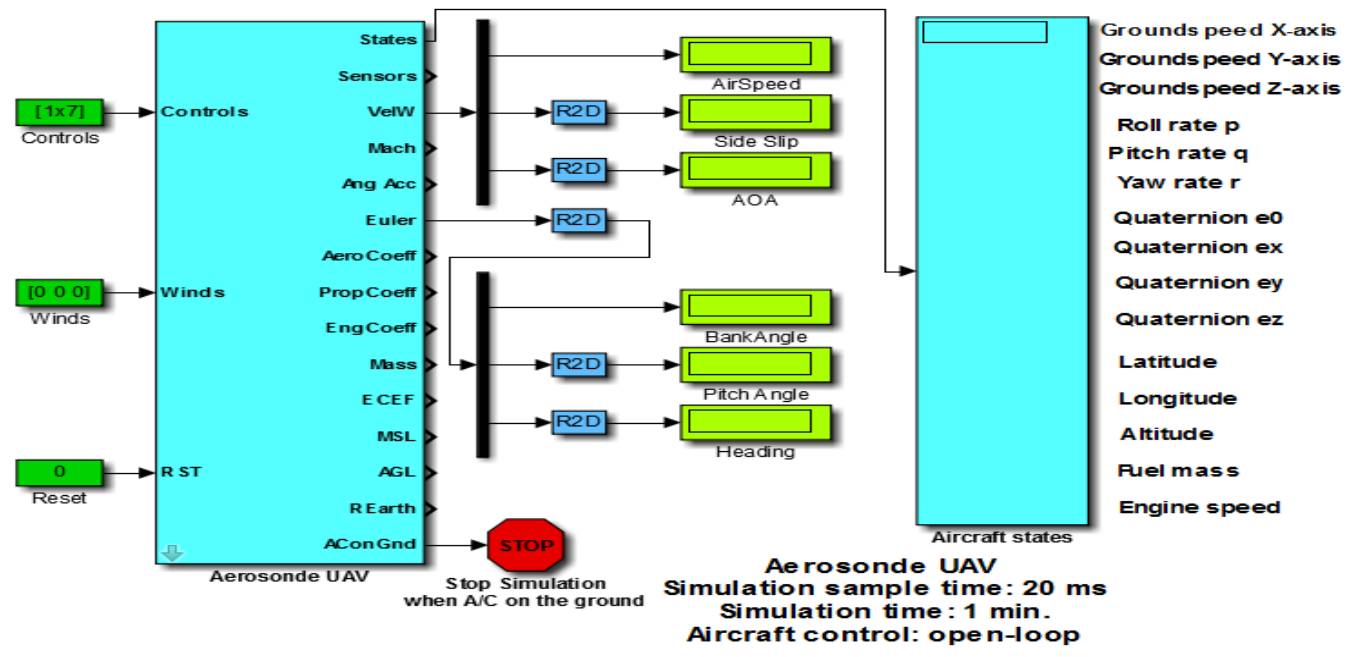

Figure 2. MATLAB simulation model in open loop 


\subsection{Aerosonde UAV dynamic model}

The UAV model is assumed to be rigid-body, with symmetric mass distribution around the aircraft's absolute coordinate system [11], based on a non-linear model described by Newton's second law and represented by 12 dynamic variables using [12]:

Force equations:

$$
\begin{aligned}
& \dot{u}=-q \cdot w+v \cdot r+x / m-g \cdot S_{\theta} \\
& \dot{v}=-q \cdot w+p \cdot w+y / m+g \cdot C_{\theta} S_{\phi} \\
& \dot{w}=-v \cdot p+p \cdot w+{ }^{z} / m+g \cdot C_{\theta} C_{\phi}
\end{aligned}
$$

Kinematic equations:

$$
\begin{aligned}
& \dot{\phi}=p+q S_{\phi} \tan \theta+r C_{\phi} \tan \theta \\
& \dot{\theta}=q C_{\phi}-r S_{\theta} \\
& \dot{\psi}=q S_{\phi} \sec \theta+r C_{\phi} \sec \theta
\end{aligned}
$$

Moment equations:

$$
\begin{aligned}
& \dot{p}=q\left(c_{1} r+c_{2} p\right)+c_{3} L+c_{4} N \\
& \dot{q}=c_{5} p \cdot r-c_{6}\left(p^{2}-r^{2}\right)+c_{7} M \\
& \dot{r}=q\left(c_{8} p-c_{2} r\right)+c_{4} L+c_{9} N
\end{aligned}
$$

Navigation equations:

$$
\begin{aligned}
& \dot{P}_{n}=u C_{\theta} C_{\psi}+v\left[S_{\varphi} S_{\theta} C_{\psi}-C_{\varphi} S_{\psi}\right]+w\left[S_{\varphi} S_{\psi}+C_{\varphi} S_{\theta} C_{\psi}\right] \\
& P_{e}=u C_{\theta} S_{\psi}+v\left[C_{\varphi} C_{\psi}+S_{\theta} S_{\varphi} S_{\psi}\right]+w\left[C_{\varphi} S_{\theta} S_{\psi}-S_{\varphi} C_{\psi}\right] \\
& \dot{h}=u S_{\theta}-v S_{\varphi} C_{\theta}-w C_{\varphi} C_{\theta}
\end{aligned}
$$

where:

$$
\begin{aligned}
& \Gamma c_{1}=I_{Z}\left(I_{Y}-I_{Z}\right)-I_{X Z}{ }^{2} \\
& \Gamma c_{2}=I_{X Z}\left(I_{X}-I_{Y}+I_{Z}\right) \\
& \Gamma c_{3}=I_{Z} \\
& \Gamma c_{4}=I_{X Z} \\
& c_{5}=\frac{\left(I_{Z}-I_{Y}\right)}{I_{X}} \\
& c_{6}=I_{X Z} / I_{Y} \\
& c_{7}=1 / I_{Y} \\
& \Gamma c_{8}=I_{X}\left(I_{X}-I_{Y}\right)+I_{X Z}{ }^{2} \\
& \Gamma=I_{X} \cdot I_{Z}-I_{X Z}{ }^{2}
\end{aligned}
$$




\subsection{Actuator modelling}

There are seven different control surfaces on the Aerosonde UAV, which are detailed below: With a downward positive indication on two flaps, ailerons, elevators, and a downward positive sign on one rudder. The aileron and elevator actuators each have a \pm 15 -degree position constraint and a \pm 40 -degree per second slew rate restriction. The Rudder, on the other hand, has a \pm 20 -degree position limit and a \pm 1.15 -degree-persecond slew rate restriction. In conjunction with the engine throttle actuator, each control surface is controlled by a separate actuator. The dynamics of the actuators are represented by a second-order transfer function, whilst the dynamics of the thrust paddles are represented by a first-order transfer function [13], [14]. The actuators utilized in this model have a transfer function as:

For elevator servo:

$$
\frac{\delta_{e}}{\delta_{e c}}=\frac{W_{n}{ }^{2}}{s^{2}+2 \zeta W_{n} s+W_{n}{ }^{2}}=\frac{(30.74)^{2}}{s^{2}+(2 \times 0.0 .509 \times 30.74) s+(30.74)^{2}}=\frac{944.95}{s^{2}+31.29 s+944.95}
$$

For throttle servo:

$$
\frac{\delta_{t}}{\delta_{t c}}=\frac{1 / T}{s+1 / T}=\frac{1 / 0.2666}{s+1 / 0.2666}=\frac{3.75}{s+3.75}
$$

where: $\delta_{e}, \delta_{t}$ are elevator and throttle deflection

$\delta_{e c}, \delta_{t c}$ are elevator and throttle command

\subsection{Sensor modelling and simulation}

2.4.1. Sensor of velocity

The first-order dynamics of the velocity sensor are as:

$$
\frac{\mathrm{V}_{\mathrm{S}}(\mathrm{S})}{\mathrm{V}(\mathrm{S})}=\frac{1}{0.03 \mathrm{~s}+1}
$$

where: $V_{s}$ : Total velocity

\subsubsection{Sensor of altitude}

The first-order dynamics of the altitude sensor are:

$$
\frac{\mathrm{H}_{\mathrm{S}}(\mathrm{S})}{\mathrm{H}(\mathrm{S})}=\frac{1}{0.025 \mathrm{~s}+1}
$$

\section{AUTOPILOT CONTROLLER DESIGN}

A reliable control system is required for a successful UAV flight route. The control system of a UAV is primarily divided into two controllers. The "Lateral Controller" is a primary controller that controls the roll angle and heading of a UAV. The "Longitudinal Controller" is the other controller that regulates the pitch angle, airspeed, and altitude of a UAV [15]. The error of the controlled UAV must be taken into account for stable flying performance. As a result, in a UAV control system, a closed-loop controller is recommended [16]. The most common closed-loop control system is a (proportional, integral, and derivative) controller [17]. Error! Reference source not found. shows how a closed-loop controller assures the stability of a UAV by removing feedback error caused by measuring and monitoring the system [18].

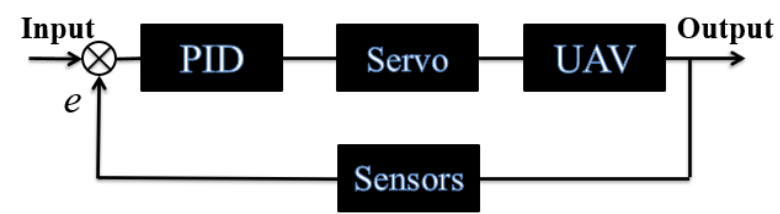

Figure 3. Control system with a closed-loop

\subsection{Classic PID controller}

A proportional, integral, and derivative component makes up a PID controller. A PID controller's transfer function is defined as [19]: 


$$
H(s)=\frac{K_{d} S^{2}+K_{p} S+K_{I}}{S}
$$

The ease with which a PID controller can be implemented is the fundamental reason for its widespread use in today's controllers. However, when a UAV is under external environmental disturbances, the PID controller requires enhanced tuning. The tuning technique for a classic PID controller is performed to eliminate the disagreement between the input and the expected output [20]. The tuning process is presented in three techniques:

a. Trial and error: depend on adding and subtracting algorithm, which is an easy way for implementing a solution, however, it takes much processing time with the unease tuning process.

b. Analytical technique: built on a mathematical model and as a result accurate results are achieved, on the contrary, this method is not applicable for highly complicated models.

c. Empirical technique: mainly based on a real model in which many parameters might be unknown, some models might be unresolved if the unknown parameters can't be predicted.

\subsection{Genetic algorithm controller}

By boosting the predicted error originating from a classic PID controller, the tuned PID controller can overcome external disturbances and dynamic changes in a system. "PID with GA" is the name given to this intelligent PID controller. The GA is a "bio-inspired algorithm" that is commonly used to optimize extremely nonlinear functions. Non-derivative techniques based on optimization approaches are used to generate the tuning process in GA [21]. The solution of a GA starts with random parameters and ends with an optimum solution based on several steps shown in Figure 4. Roll stabilizer. The tuning process in Figure 4. Roll stabilizer begins with the formation of the first population, with the fitness function selecting the fittest individuals to activate the next generation's population. The chosen individuals (parents) were collected in a mating pool, where some genes from each parent were exchanged. This is referred to as "cross-over," and it occurs when new "offspring" are produced. The probability of cross-over $\left(P_{c r}\right)$ varies between $(0.6$ to 0.9$)$. The new generation has been adjusted slightly to offer improved and optimum results. This is referred to as a mutation, with a probability $\left(P_{m u}\right)$ ranging from $(0.01$ to 0.001$)$. If the output result satisfies convergence, optimization has been accomplished; if not, the process is repeated until optimization has been achieved [22].

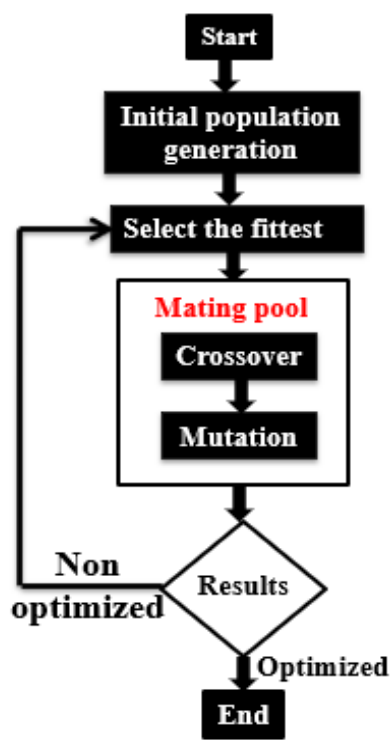

Figure 4. Roll stabilizer

\subsection{Lateral autopilot}

The lateral flight controller is used to regulate the UAV's lateral and directional motion. The lateral flight controller design is primarily concerned with navigating the UAV around the trim state, which is assumed in both lateral and directional senses [23]. According to transient analysis of aircraft natural motion, the spiral mode (which is based on the roll, yaw, and side velocity states) is unbalanced. A roll stabilizer loop is incorporated with a PID controller in the lateral flight controller as illustrates in Figure 5. 


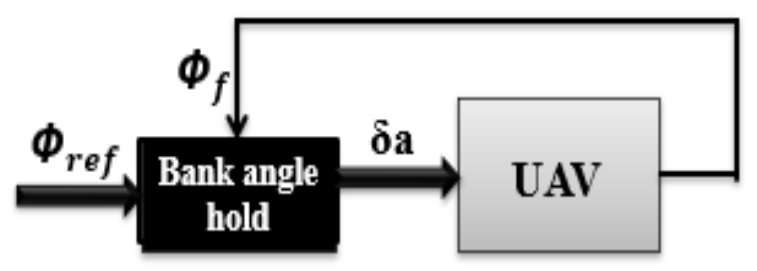

Figure 5. GA flowchart

\subsubsection{Roll stabilizer}

The unbalanced roll moment generated from engine thrust will excite the spiral mode and permit the aircraft to settle in a constant bank angle turn the roll stabilizer is designed to stabilize the spiral mode by adding what is called Wing Leveler. PID controller, which, implements this, gains are tuned using Simulink response optimization software (SROS) with pattern search optimization technique using GA method [24]. SROS includes a graphical user interface (GUI) to aid in the design of control systems. The signal constraint block is attached to the output of the Simulink block diagram for optimizing the PID gains of the roll loop for reference tracking specifications before beginning the optimization using SROS for the system. In relation to kp_phi, ki_phi, and kd_phi, the obtained PID gains were $0.6234,0.6829$, and zero, respectively. The designed controller provides an overall stabilization to roll dynamics by ensuring the value of the closed-loop bank angle to be zero as shown in Figure 6.

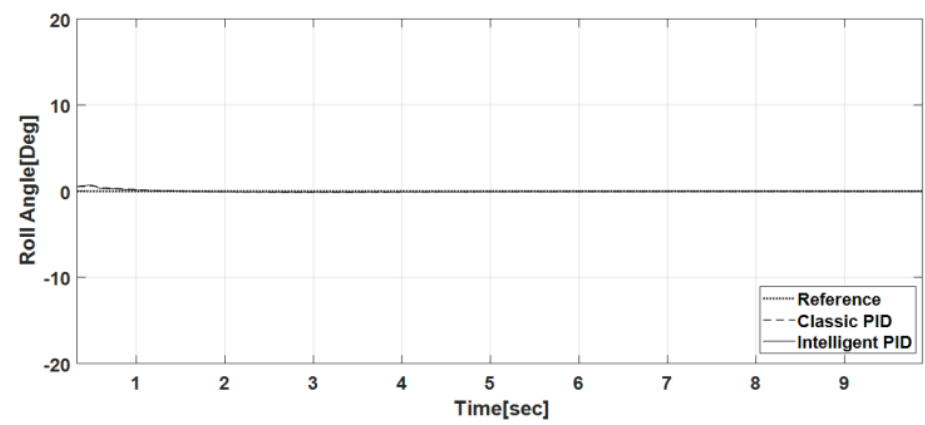

Figure 6. Closed-loop bank angle

\subsection{Longitudinal autopilot}

As indicated in Figure 7, two control loops, altitude and airspeed, are used to build the longitudinal flight controller [25]. Three longitudinal autopilot scenarios are carried out and explained in section 4.

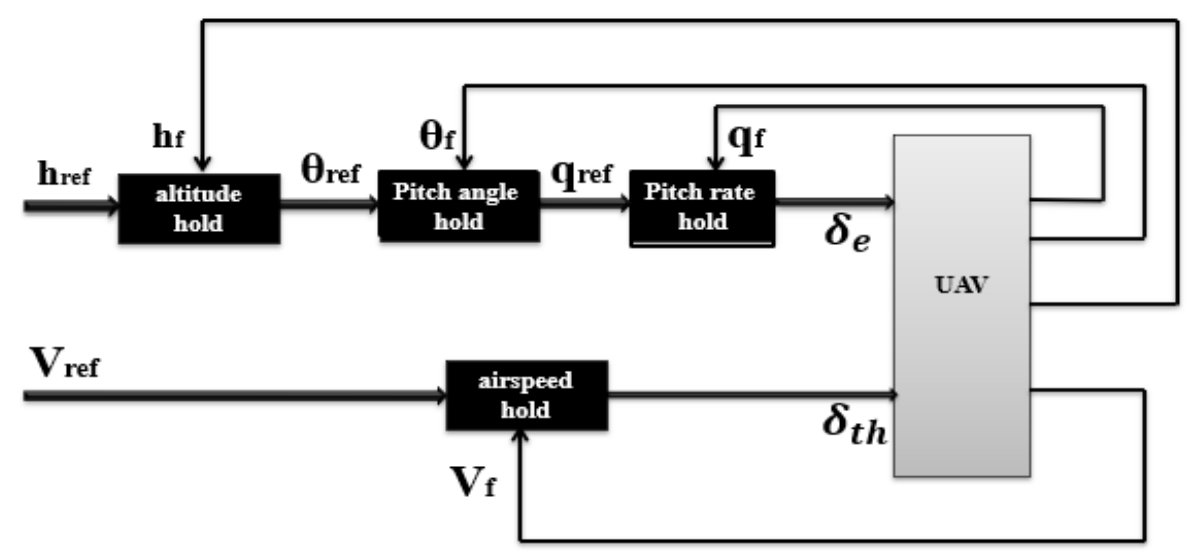

Figure 7. Longitudinal motion autopilot 


\section{RESULTS OF THE SIMULATION}

\subsection{First simulation scenario}

Figure 8 shows the step response of altitude with constant speed under normal conditions. Figure 9 shows a step response of velocity with constant altitude. The PID controller with GA has better temporal responsiveness than the classical PID, as seen in both Figures 8-9.

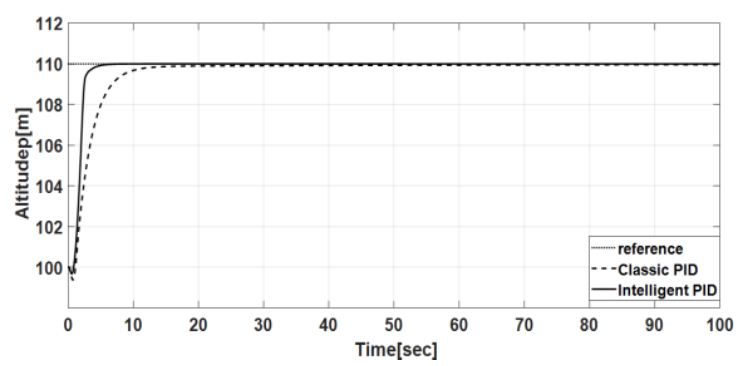

Figure 8. The response of step altitude

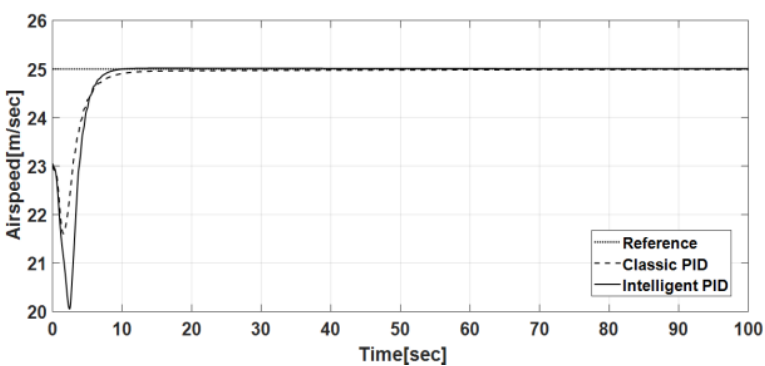

Figure 9. The response of step airspeed

\subsection{Second simulation scenario (reference tracking)}

Figure 10 shows a comparison between the classic PID controller and the PID controller with GA at constant airspeed with a reference altitude. The classic PID controller's results are in good agreement with the PID controller with GA. Any change in altitude affects the change in speed in a reciprocal manner. Figure 11 indicates that there is no difference between the classic PID controller and the PID controller with GA in terms of reference airspeed. The altitude values are slightly varied for any speed adjustment.

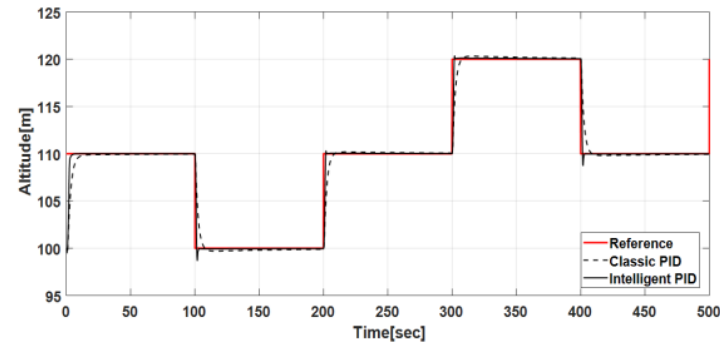

Figure 10. Altitude response at a constant airspeed

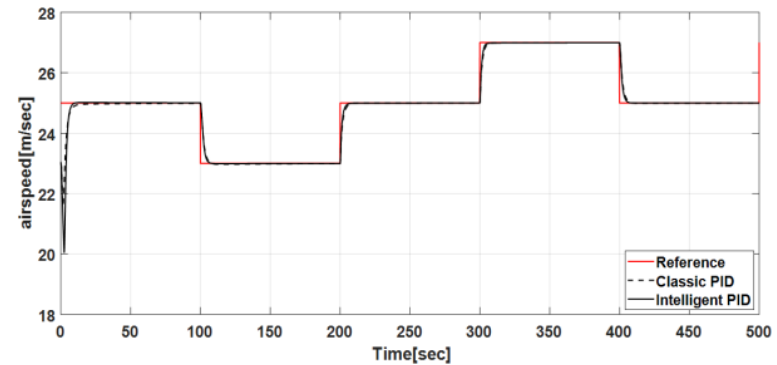

Figure 11. Airspeed response at a constant altitude

\subsection{Third simulation scenario}

In this scenario, external wind disturbance and noise are taken into consideration. During the simulation time, both wind disturbance and noise are introduced to the UAV model, as seen in Figure 12 and Figure 13. For certain values, the controllers provide a consistent reaction; but, beyond these values, instability arises. The PID controller with GA, on the other hand, has better responsiveness.

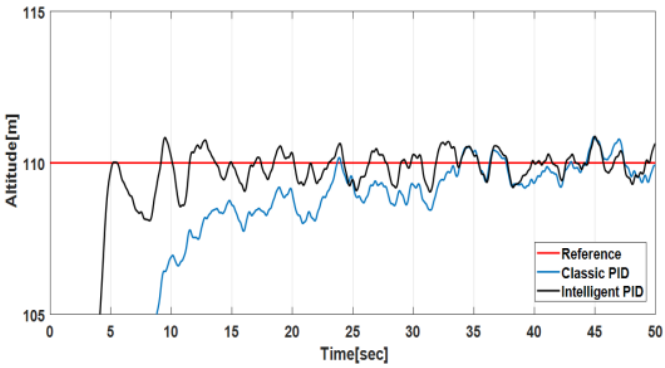

Figure 12. The impact of wind on altitude response

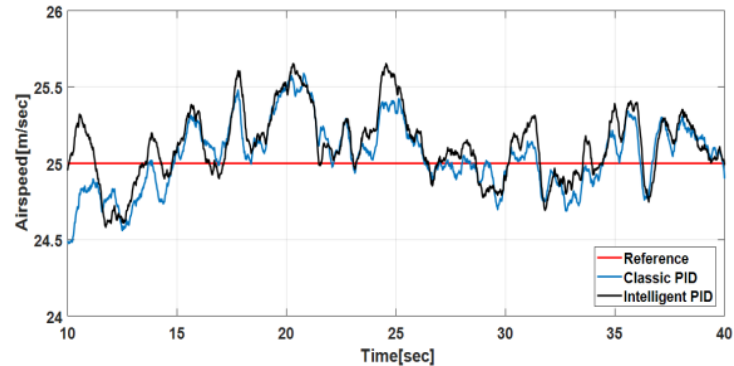

Figure 13. The impact of wind on airspeed response 


\section{CONCLUSION}

The aerosonde UAV's dynamic model equations are represented in this paper. The attitude and altitude control of the Aerosonde UAV is controlled by an intelligent PID controller. The proposed controller is simulated under normal conditions as well as in the presence of external wind disturbance and noise, and the results are compared to those of a traditional PID controller. Simulation findings show that the system is more robust and durable than a traditional PID, moreover, the proposed controller parameters are proved to be optimized using a GA.

\section{REFERENCES}

[1] J. Xu, Q. Guo, L. Xiao, Z. Li, and G. Zhang, "Autonomous Decision-Making Method for Combat Mission of UAV based on Deep Reinforcement Learning," in 2019 IEEE 4th Advanced Information Technology, Electronic and Automation Control Conference (IAEAC), 2019, pp. 538-544, doi: 10.1109/IAEAC47372.2019.8998066.

[2] G. Zogopoulos-Papaliakos, G. C. Karras, and K. J. Kyriakopoulos, "A Fault-Tolerant Control Scheme for FixedWing UAVs with Flight Envelope Awareness,” J. Intell. Robot. Syst., vol. 102, no. 2, pp. 1-33, 2021, doi: 10.1007/s10846-021-01393-3.

[3] M. K. Elbaioumy, M. M. Elkhatib, and A. E. Khalifa, "Modelling and Simulation of Surface to Surface Missile General Platform.," Adv. Mil. Technol., vol. 13, no. 2, 2018, pp. 277-290, doi: 10.3849/aimt.01236.

[4] J. M. Peschel and R. R. Murphy, "Human interfaces in micro and small unmanned aerial systems," Handb. Unmanned Aer. Veh., pp. 2389-2403, 2015, doi: 10.1007/978-90-481-9707-1_127.

[5] A. Aghaeeyan, F. Abdollahi, and H. A. Talebi, "UAV-UGVs cooperation: With a moving center based trajectory," Rob. Auton. Syst., vol. 63, pp. 1-9, 2015, doi: 10.1016/j.robot.2014.10.005.

[6] Y. Fan, M. Ding, and Y. Cao, "Vision algorithms for fixed-wing unmanned aerial vehicle landing system," Sci. China Technol. Sci., vol. 60, no. 3, pp. 434-443, 2017, doi: 10.1007/s11431-016-0618-3.

[7] R. Anandan and K. Kalaivani, "A Precise Model of Insect Flight Vitality and Development of Unmanned Micro Aerial Vehicle," J. Inst. Eng. Ser. B, pp. 1-12, 2021, doi: 10.1007/s40031-021-00619-0.

[8] R. Socas, S. Dormido, and R. Dormido, "Event-based control strategy for the guidance of the Aerosonde UAV," in 2015 European Conference on Mobile Robots (ECMR), 2015, pp. 1-6, doi: 10.1109/ECMR.2015.7324213.

[9] I. d. M. Esper and P. F. Ferreira Rosa, "Heading Controller for a Fixed Wing UAV with Reduced Control Surfaces Based on ANFIS," 2015 IEEE International Conference on Dependable Systems and Networks Workshops, 2015, pp. 118-123, doi: 10.1109/DSN-W.2015.27.

[10] A. Sarhan and S. Qin, "Robust Adaptive Flight Controller for UAV Systems," 2017 4th International Conference on Information Science and Control Engineering (ICISCE), 2017, pp. 1214-1219, doi: 10.1109/ICISCE.2017.252.

[11] B. Zhu, J. Zhu, and Q. Chen, "A bio-inspired flight control strategy for a tail-sitter unmanned aerial vehicle," Sci. China Inf. Sci., vol. 63, pp. 1-10, 2020, doi: 10.1007/s11432-019-2764-1.

[12] S. Zhao, X. Wang, H. Chen, and Y. Wang, "Cooperative Path Following Control of Fixed-wing Unmanned Aerial Vehicles with Collision Avoidance," J. Intell. Robot. Syst., vol. 100, no. 3, pp. 1569-1581, 2020, doi: 10.1007/s10846-020-01210-3.

[13] M. Korany, A. M. Youssef, and G. El-sheikh, "Comparative Synthesis Of UAV Flight Control In Presence Of Actuators And Sensors Dynamics," in The 9th international conference on electrical engineering, pp. 1-8, 2014, doi: 10.21608/ICEENG.2014.30458.

[14] A. Elbatal, M. M. Elkhatib and A. M. Youssef, "Intelligent Autopilot Design Based on Adaptive Neuro -Fuzzy Technique and Genetic Algorithm," 2020 12th International Conference on Electrical Engineering (ICEENG), 2020, pp. 377-382, doi: 10.1109/ICEENG45378.2020.9171702.

[15] F. Lin, H. Duan and X. Qu, "PID control strategy for UAV flight control system based on improved genetic algorithm optimization," The 26th Chinese Control and Decision Conference (2014 CCDC), 2014, pp. 92-97, doi: 10.1109/CCDC.2014.6852124.

[16] S. G. Mathisen, F. S. Leira, H. H. Helgesen, K. Gryte, and T. A. Johansen, “Autonomous ballistic airdrop of objects from a small fixed-wing unmanned aerial vehicle," Auton. Robots, vol. 44, no. 5, pp. 859-875, 2020, doi: 10.1007/s10514-020-09902-3.

[17] T. Muhammad Hassan et al., "PID based controller design for attitude stabilization of Quad-rotor," Australian Journal of Basic and Applied Sciences, vol. 8, no.4, pp. 1-5, 2014.

[18] H. Noshahri and H. Kharrati, "PID controller design for unmanned aerial vehicle using genetic algorithm," 2014 IEEE 23rd International Symposium on Industrial Electronics (ISIE), 2014, pp. 213-217, doi: 10.1109/ISIE.2014.6864613.

[19] T. Espinoza-Fraire, A. Dzul, F. Cortés-Martínez, and W. Giernacki, "Real-time implementation and flight tests using linear and nonlinear controllers for a fixed-wing miniature aerial vehicle (MAV)," Int. J. Control. Autom. Syst., vol. 16, no. 1, pp. 392-396, 2018, doi: 10.1007/s12555-016-0590-9.

[20] H. A. de Oliveira and P. F. F. Rosa, "Adaptive genetic neuro-fuzzy attitude control for a fixed wing UAV," 2017 IEEE International Conference on Industrial Technology (ICIT), 2017, pp. 726-731, doi: 10.1109/ICIT.2017.7915449.

[21] Y. Chen, S. Liu, C. Xiong, Y. Zhu and J. Wang, "Research on UAV Flight Tracking Control Based on Genetic Algorithm optimization and Improved bp Neural Network pid Control," 2019 Chinese Automation Congress (CAC), 2019, pp. 726-731, doi: 10.1109/CAC48633.2019.8996179. 
[22] H. A. de Oliveira and P. F. Ferreira Rosa, "Genetic neuro-fuzzy approach for unmanned fixed wing attitude control," 2017 International Conference on Military Technologies (ICMT), 2017, pp. 485-492, doi: 10.1109/MILTECHS.2017.7988808.

[23] M. A. A. Osman, H. E. A. Abdalla and M. O. Nawari, "Lateral and Longitudinal Controllers Design for a Fixed Wing UAV," 2019 International Conference on Computer, Control, Electrical, and Electronics Engineering (ICCCEEE), 2019, pp. 1-6, doi: 10.1109/ICCCEEE46830.2019.9071049.

[24] A. A. Najm, A. T. Azar, I. K. Ibraheem, and A. J. Humaidi, "A nonlinear PID controller design for 6-DOF unmanned aerial vehicles," in Unmanned Aerial Systems, Elsevier, 2021, pp. 315-343, doi: 10.1016/B978-0-12820276-0.00020-0.

[25] M. Kamel, T. Stastny, K. Alexis, and R. Siegwart, "Model predictive control for trajectory tracking of unmanned aerial vehicles using robot operating system," in Robot operating system (ROS), Springer, 2017, pp. 3-39, doi: 10.1007/978-3-319-54927-9_1.

\section{BIOGRAPHIES OF AUTHORS}

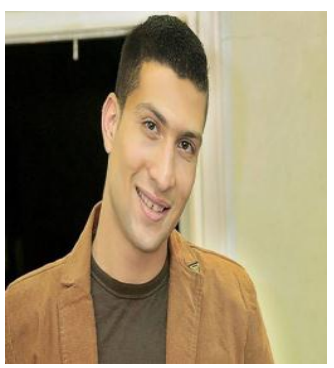

Ahmed Elbatal earned a B.Sc. in Electrical Engineering from Cairo's Military Technical College in 2015. He has been working as a researcher in the field of electrical engineering for 5 years and now he is studying for the fulfilment of M. Sc. in flight control engineering in Military Technical College, Egypt.

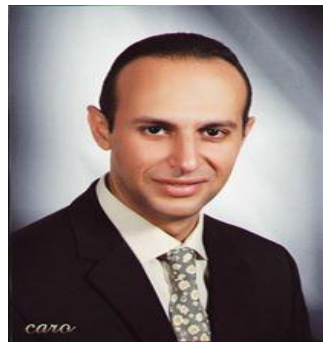

Prof. Ahmed Medhat Youssef got B.Sc. and M.Sc. degrees in electrical engineering from MTC in Cairo, Egypt in 1993 and 1998. In 2004, he obtained his Ph. D. from "The University of Strathclyde", UK. In 2013, he was promoted to Associate Professor of Electrical Engineering. He is now the head of Military Technical College's Aircraft Electric Equipment Department in Cairo, Egypt.

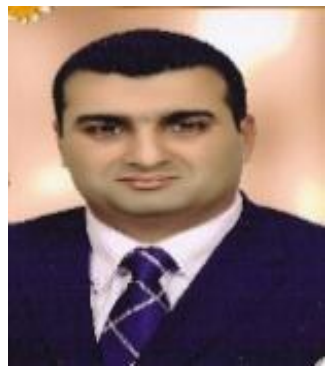

Prof. Mohamed M. Elkhatib is IEEE Senior Member. In 1994 and 1999, he received his B.Sc. and M.Sc. degrees from MTC in Cairo, Egypt. In December 2007, he got his PhD from the University of Strathclyde, UK. He was a postdoctoral visiting scholar at Ohio State University in 2016. He has worked as a professor in the EEE Department at Military Technical College. He currently serves as Co-Editor-in-Chief for the EJMTC Journal. 